\title{
Are sleep duration, midpoint of sleep and sleep quality associated with dietary intake among Bavarian adults?
}

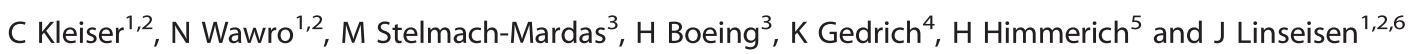

BACKGROUND/OBJECTIVES: Only few epidemiologic studies examined sleep characteristics in relation to dietary behaviour. Our aim was to analyse associations of sleep duration, midpoint of sleep and sleep quality with dietary intake among the Bavarian population.

SUBJECTS/METHODS: Within the cross-sectional Bavarian Food Consumption Survey II, 1050 subjects aged 13-81 years were recruited. Dietary intake was assessed with three 24-h dietary recalls by telephone (EPIC-Soft). In our study, 814 participants aged 18 years or older, who completed at least two 24-h dietary recalls and who had complete and plausible information on sleep characteristics were analysed. Dietary intake was described by the consumption of main food groups, energy-proving nutrients and energy intake. Sleep was measured by the Pittsburgh Sleep Quality Index Questionnaire, from which categories of self-reported usual sleep duration in half-h-steps per night, midpoint of sleep and overall sleep quality were derived.

RESULTS: Sleep duration was associated with intake of non-alcoholic beverages $(P<0.01)$, carbonated beverages $(P=0.04)$, water $(P=0.04)$ and coffee/black tea $(P=0.01)$ with higher intake among short duration sleepers. No association was found between the consumption of other main food groups, energy-proving nutrients or total daily energy intake and sleep duration. Midpoint of sleep was associated with intake of carbonated beverages $(P=0.02$, highest intake among subjects with early midpoint of sleep).

No association between sleep quality and dietary intake was detected.

CONCLUSIONS: Our findings demonstrate only specific associations between sleep characteristics and dietary intake, and mainly sleep duration was associated with beverage intake.

\section{INTRODUCTION}

Recent findings from large epidemiologic studies provide evidence that sleep duration is associated with the incidence of diet-related chronic diseases such as obesity, type 2 diabetes mellitus, hypertension or the metabolic syndrome and mortality. ${ }^{1-5}$ The mechanisms explaining these observations are complex and are still under discussion. The influence of sleep on dietary behaviour and energy metabolism might be one link explaining these diet-health relations.

Experimental studies have shown that subjects under sleep restriction had increased food, energy and fat intake and consumed larger food portions and more calories from snacks. ${ }^{6-11}$ Some of these studies further support the hypothesis that sleep has an impact on metabolic and hormonal changes. In case of sleep deprivation, levels of the appetite-stimulating hormone ghrelin were increased, whereas levels of the appetitesuppressing hormone leptin were reduced. ${ }^{12,13}$ Other studies could not support these findings, ${ }^{7,10,11}$ suggesting that the mechanism of how sleep deprivation changes food and energy intake might be more complex.

Only few epidemiological studies examined the association between sleep characteristics and dietary behaviour, mostly describing a possible influence of sleep duration on specific aspects of dietary intake among cross-sectional populations but with different results. ${ }^{14-18} \mathrm{~A}$ review of cross-sectional studies suggests that short sleep duration is related to higher intake of total energy and fat. In contrast, there is only restricted evidence for the association between short sleep duration and lower intake of fruit. ${ }^{19}$ Very few studies examined the association between the human chronotype, for example, the individual affinity to sleep at a certain time during a 24-h period, and dietary behaviour. Chronotype is associated with intakes of certain nutrients and foods and in particular the evening chronotype is related to unfavourable dietary habits. ${ }^{20,21}$ Two recent studies analysed whether sleep quality is related to food and nutrient intake. The results show that the intake of several nutrients and foods was associated with different aspects of sleep quality. 22,23

The findings of these existing studies describe a broad range of aspects regarding the associations between sleep characteristics and dietary behaviour suggesting that there is a relationship between sleep and diet. However, the results differ and are not consistent. The aim of our study was to examine whether there is an association between sleep duration, midpoint of sleep or sleep quality and food intake and whether these sleep characteristics are related differently to food intake among the Bavarian population in Germany.

${ }^{1}$ Helmholtz Zentrum München, Institute of Epidemiology II, Neuherberg, Germany; ${ }^{2}$ German Center for Diabetes Research (DZD e.V.), Neuherberg, Germany; ${ }^{3}$ Department of Epidemiology, German Institute of Human Nutrition, Nuthetal, Germany; ${ }^{4}$ Nutritional Physiology, TUM School of Life Sciences Weihenstephan, Technische Universität München, Freising, Germany; ${ }^{5}$ Department of Psychological Medicine, Institute of Psychiatry, Psychology \& Neuroscience (loPPN), King's College London, Denmark Hill, London, UK and ${ }^{6}$ Universitary Center of Health Sciences at Klinikum Augsburg - UNIKA-T, Institute of Epidemiology, Augsburg, Germany. Correspondence: Professor J Linseisen, Helmholtz Zentrum München, Institute of Epidemiology II, Ingolstädter Landstr. 1, 85764 Neuherberg, Germany,

E-mail: j.linseisen@helmholtz-muenchen.de 


\section{MATERIALS AND METHODS}

Study design

The Bavarian Food Consumption Survey II (BVS II) is a cross-sectional study representative for the Bavarian population investigating dietary and lifestyle habits. Between September 2002 and June 2003, 1050 Germanspeaking subjects aged 13-80 years were recruited following a three-stage random sampling procedure. This recruitment procedure included the selection of 42 communities as so-called sampling points (stratified by county and community characteristics), a random walk (every third household) with a given start address and a random selection of one household member who meets the selection criteria. At baseline, data on lifestyle, sleep characteristics, socioeconomic and health status were collected using a computer-assisted personal interview. Within the following 2 weeks, dietary intake was assessed by three standardised and computer-assisted 24-h dietary recalls (24-h DR) by telephone ( 2 weekdays, 1 weekend day) conducted by trained interviewers using the software EPIC-Soft. ${ }^{24,25}$ All adult study subjects ( $\geqslant 18$ years) who had completed at least one 24-h DR $(n=879)$ were invited to their nearest health office for blood sampling and standardised anthropometric measurements within 6 weeks after recruitment. The overall participation rate in the study was $71 \%$. Written informed consent was obtained from all subjects. The study was approved by the ethics committee of the Bavarian State Medical Association (Protocol No. 2111). For the analyses presented below, we used data from subjects who were at least 18 years old and completed at least two 24-h DR $(n=848)$. Of those, we excluded 34 subjects because of missing or implausible information on sleep characteristics, resulting in $n=814$ subjects included in our analyses.

\section{Sleep characteristics}

To assess sleep duration, midpoint of sleep and sleep quality, the Pittsburgh Sleep Quality Index (PSQI) questionnaire was used, which is described in detail elsewhere. ${ }^{26}$ Briefly, the PSQI consists of seven components describing the following sleep characteristics: subjective sleep quality, sleep latency, sleep duration, habitual sleep efficiency, sleep disturbance, use of sleep medication and daytime dysfunction. For each of the components, the score ranges from 0 to 3 . Therefore, the PSQI as sum of those scores can range from 0 to 21. In the present study, a shortened version of the PSQI was applied as part of the personal interview, consisting of 10 questions covering all 7 components with an overall range from 0 to 21 . A lower total score represents higher sleep quality.

To analyse sleep duration, we used the question 'During the past 4 weeks, how many hours of actual sleep did you get at night? (This may be different than the number of hours you spend in bed.)'. Answers were given in half-h-steps and grouped into five sleep duration categories $(<6,6-6.5,7,7.5-8$ and $>8 \mathrm{~h})$.

The midpoint of sleep on weekdays was calculated as the midpoint between time falling asleep and rise time (time falling asleep+(sleep duration/2)). Subjects were classified into tertiles of midpoint of sleep from early (T1) to medium (T2) and late (T3).

To assess sleep quality, the PSQI was calculated. Based on a total score from 0 to 21, subjects were classified as either poor (PSQI $>5$ ) or good sleepers (PSQI $<=5$ ), as defined by Buysse et al. ${ }^{26}$

\section{Food and nutrient intake}

The 24-DR interview was performed by telephone using the standardised software EPIC-SOFT. The structure of the interview was described in detail elsewhere. ${ }^{24}$ Briefly, the 24-DR is divided into the following four sections: general non-dietary information, a quick list of foods consumed the day before the interview (foods are recorded per meal), a detailed description and quantification of the consumed foods and quality control questions. In the present study, data from the 24-h DR were linked with the German Nutrient Data Base BLS, version II.3 (BgVV, Berlin, Germany) to estimate nutrient intake. Data from at least two 24-h DR were used and weighted for weekday or weekend day to calculate the mean amount of daily food and nutrient intake per subject per day (g/day, gram per day). Analogously, the mean daily energy intake (kcal/day, kilocalories per day) was derived. The weighting factor for each of the two weekdays was $2.5 / 7$, and the factor for the weekend day was 2/7. For 15 subjects, only two 24-hour recalls were available. For those subjects, a weighting factor of $5 / 7$ was used for the one weekday.
Assessment of covariates

Covariates used in the analyses were collected during the personal interview and included sex, subject's age in years and body mass index (BMI). The level of education was included as categorical variable grouping subjects into those with 'at least 12 years of education' and those with 'less than 12 years of education'. Smoking status with the standard categories of 'smoker', 'ex-smoker' and 'non-smoker' as well as weekly physical activity (h/week), categorised in 'at least $2 \mathrm{~h} /$ week' and 'less than $2 \mathrm{~h} /$ week', and daily television (TV) and/or computer (PC) use in hours per day were included. The season of assessment was used as a proxy for daylight duration and classified into 'spring' (March 21st-June 20th), 'summer' (June 21st-Sept 20th), 'autumn' (Sept 21st-Dec 22nd) and 'winter' (dec 23rd-March 20th), according to the date the personal interview was conducted.

\section{Statistical analysis}

All statistical analyses were performed using SAS software package version 9.3 (SAS Institute, Cary, NC, USA).

Main characteristics of the study population were described separately for sleep duration, midpoint of sleep and sleep quality. Frequency and percentage or arithmetic means and s.d.'s were calculated stratified by sleep characteristic. The $x^{2}$-test for categorical variables and a one-way ANOVA for continuous variables were used to test for differences in sleep characteristic by covariates.

We performed an exploratory analysis to assess whether sleep duration midpoint of sleep or sleep quality are related to daily food intake. For each of the main food groups, adjusted means of daily intake and 95\% confidence intervals were calculated, stratified by the relevant sleep characteristic.

Adjusted means of daily intake of the main food groups by sleep characteristic groups were calculated using the least squares means statement in the generalized linear models procedure, taking into account covariates known to be related with sleep or dietary intake and which were available in the study. These covariates are age, sex, BMI, education, smoking, physical activity, TV/PC use, season and energy intake, as described above. Analogously, adjusted means of daily energy intake took into account age, sex, BMI, education, smoking, physical activity, TV/PC use and season.

The differences between the adjusted means by sleep characteristic groups were determined using the PDIFF option in the least squares means statement that returns $P$-values for multiple pairwise comparisons of the adjusted means (Student's $t$-test). For these $P$-values, a Bonferroni adjustment was applied to correct for multiple testing. The F-test in the generalized linear models procedure statement was used to test for a general effect of the sleep characteristic.

A priori, we examined interactions between sleep characteristics and sex, age and BMI with regard to food intake. We included interaction terms (sleep*sex, sleep*age or sleep*BMI) for each sleep characteristic variable in every single GLM procedure (for example, 'daily food intake' = sleep duration+sex+sleep duration*sex+age+BMl+education+smoking+physical activity+TV/PC use+season+ energy intake). As there were only very few significant interactions between sleep characteristics and sex, age and $\mathrm{BMI}$, we present our results for the total study population. The results of analyses stratified for sex, age and BMI can be found as Supplementary information.

Furthermore, we performed sensitivity analyses by the exclusion of under-reporters ( $n=78,30$ men, 48 women). These were defined by a ratio of caloric intake (kcal) to basal metabolic rate (kcal) of $<0.8{ }^{27}$

As the main aim of the study was to describe dietary intake in the Bavarian population, no power calculation for testing the association between sleep characteristics and diet was conducted. A posterior conducted power calculation showed that with the given sample size of $N=814$, an $a$ of $5 \%$ and a power of $80 \%$, even very small mean differences in food group intake between the five sleep duration groups can be detected.

\section{RESULTS}

Descriptive statistics of sleep duration, midpoint of sleep and sleep quality stratified by main subject characteristics are shown in Tables $1 a$ and $b$. Sleep duration did not differ considerably according to sex, age, BMI, physical activity, smoking and season, but it was associated with education and TV/PC use. Both short 
and long duration sleepers more often showed a lower education and had a higher TV/PC use. There was no trend across sleep duration categories (Table 1a). Furthermore, midpoint of sleep showed differences according to sex, TV/PC use and smoking. Early midpoint of sleep was more prevalent among women. Subjects with late midpoint of sleep showed the highest TV/PC use and were more often current smokers than those with early or medium midpoint of sleep. No differences in midpoint of sleep were observed with respect to age, BMI, education, physical activity and season (Table 1b). Sleep quality was found to vary with age, BMI $\left(\mathrm{kg} / \mathrm{m}^{2}\right)$, physical activity, TV/PC use and smoking Subjects with a good sleep quality were younger, had a lower BMI were more physically active, had less TV/PC use and were less often smokers than subjects with poor sleep quality. Sleep quality did not differ between men and women as well as with BMI groups, education and season (Table $1 \mathrm{~b}$ ). Table 2 shows the adjusted means of daily food intake of the total study population stratified by sleep duration categories. Sleep duration is associated with intake of non-alcoholic beverages $(P<0.01)$, that is, higher intake amounts are found among short duration sleepers. For carbonated beverages, water and coffee/black tea the highest intake is observed among subjects sleeping less than $6 \mathrm{~h}$. Short duration sleepers $(<6 \mathrm{~h})$ showed a significantly higher intake of non-alcoholic beverages than subjects of all other sleeping groups did $(P<0.05)$. Furthermore, short duration sleepers had significantly higher intake of coffee/black tea compared with those sleeping $7 \mathrm{~h}$ and those sleeping more than $8 \mathrm{~h}(P<0.05)$. Sleep duration was not associated with the intake of other main food groups, energy-providing nutrients and energy intake.

Adjusted means of daily food intake of the total study population stratified by the midpoint of sleep (in tertiles) are shown in Table 3. Midpoint of sleep was only significantly associated with the intake of carbonated beverages $(P=0.02)$, where the highest intake was observed for subjects with an early midpoint of sleep. As shown in Table 4, there was no significant association between overall sleep quality and food intake.

The sensitivity analyses showed that the results did not differ considerably when under-reporters were excluded. Furthermore, the $P$-values for multiple pairwise comparisons of the adjusted means are corrected for multiple testing using the Bonferroni adjustment, strengthening the robustness of our findings.

\section{DISCUSSION}

In line with previous studies, we identified associations between sleep duration and beverage consumption. For example, crosssectional data from 15.199 NHANES participants showed that in comparison to average duration sleepers, sugar and caffeine intakes were higher in short duration sleepers, which is comparable to our results concerning intake of coffee/tea and carbonated beverages. Furthermore, there was no difference in energy intake across sleep duration categories. ${ }^{14}$ Another analysis of NHANES data showed that amongst others tap water, total carbohydrate and alcohol were associated with sleep duration. ${ }^{15}$ However, the

\begin{tabular}{|c|c|c|c|c|c|c|c|}
\hline & \multirow[t]{2}{*}{ Total sample } & \multicolumn{6}{|c|}{ Sleep duration categories } \\
\hline & & $<6 h$ & $6-6.5 h$ & $7 h$ & $7.5-8 h$ & $>8 h$ & $\mathrm{P}^{\mathrm{a}}$ \\
\hline $\mathrm{N}(\%)$ & 814 & $71(8.7)$ & $162(19.9)$ & $254(31.2)$ & 265 (32.6) & $62(7.6)$ & \\
\hline \multicolumn{8}{|l|}{$\operatorname{Sex}(\mathrm{N}(\%))$} \\
\hline Men & $327(40.2)$ & $30(42.3)$ & $69(42.6)$ & $101(39.8)$ & $99(37.4)$ & $28(45.2)$ & 0.73 \\
\hline Women & $487(59.8)$ & $41(57.7)$ & $93(57.4)$ & $153(60.2)$ & $166(62.6)$ & $34(54.8)$ & \\
\hline Age (mean \pm s.d.) & $48.0 \pm 15.4$ & $49.6 \pm 16.8$ & $47.6 \pm 14.7$ & $46.9 \pm 14.2$ & $48.6 \pm 15.4$ & $48.7 \pm 19.6$ & 0.62 \\
\hline \multicolumn{8}{|l|}{ BMl groups: $\mathrm{N}(\mathrm{N}(\%))$} \\
\hline Underweight & $18(2.2)$ & $4(5.6)$ & $5(3.1)$ & $3(1.2)$ & $4(1.5)$ & $2(3.2)$ & 0.37 \\
\hline Normal weight & $337(41.4)$ & $24(33.8)$ & $66(40.7)$ & $104(40.9)$ & $118(44.5)$ & $25(40.3)$ & \\
\hline Overweight & $296(36.3)$ & $23(32.4)$ & $60(37.0)$ & $97(38.2)$ & $90(34.0)$ & $26(41.9)$ & \\
\hline Obese & $163(20.0)$ & $20(28.2)$ & $31(19.1)$ & $50(19.7)$ & $53(20.0)$ & $9(14.5)$ & \\
\hline BMI $\left(\mathrm{kg} / \mathrm{m}^{2}\right)$ & $26.5 \pm 5.1$ & $26.7 \pm 5.7$ & $26.5 \pm 5.9$ & $26.7 \pm 4.8$ & $26.3 \pm 4.8$ & $25.8 \pm 4.5$ & 0.67 \\
\hline \multicolumn{8}{|l|}{ Education (N (\%)) } \\
\hline$<12$ years & $609(78.8)$ & $60(84.5)$ & $113(69.8)$ & $194(76.4)$ & $191(72.1)$ & $52(83.9)$ & 0.03 \\
\hline$\geqslant 12$ years & $177(21.7)$ & $10(14.1)$ & $43(26.5)$ & $54(21.3)$ & $64(24.2)$ & $6(9.7)$ & \\
\hline Missing & $28(3.4)$ & $1(1.4)$ & $6(3.7)$ & $7(2.8)$ & $10(3.8)$ & $4(6.5)$ & \\
\hline \multicolumn{8}{|l|}{ Physical activity (N (\%)) } \\
\hline$\geqslant 2 \mathrm{~h} /$ week & $448(55.0)$ & $31(43.7)$ & $90(55.6)$ & $144(56.7)$ & $153(57.8)$ & $30(48.4)$ & 0.21 \\
\hline$<2 \mathrm{~h} /$ week & $366(45.0)$ & $40(56.3)$ & $72(44.4)$ & $110(43.3)$ & $112(42.3)$ & $32(51.6)$ & \\
\hline TV and PC use (h/day) & $2.5 \pm 2.1$ & $3.6 \pm 2.6$ & $3.0 \pm 2.1$ & $2.6 \pm 2.0$ & $2.5 \pm 1.9$ & $3.1 \pm 2.3$ & $<0.01$ \\
\hline \multicolumn{8}{|l|}{ Smoking (N (\%)) } \\
\hline Current smoker & $217(26.7)$ & $20(28.2)$ & $45(27.8)$ & $67(26.4)$ & $68(25.7)$ & $17(27.4)$ & 0.18 \\
\hline Ex-smoker & $174(21.4)$ & $16(22.5)$ & $47(29.0)$ & $42(16.5)$ & $55(20.8)$ & $14(22.6)$ & \\
\hline Non-smoker & $422(51.8)$ & $35(49.3)$ & $70(43.2)$ & $144(56.7)$ & $142(53.6)$ & $31(50.0)$ & \\
\hline Missing & $1(0.1)$ & & & $1(0.4)$ & & & \\
\hline \multicolumn{8}{|l|}{ Season (N (\%)) } \\
\hline Spring & $258(31.7)$ & $22(31.0)$ & $54(33.3)$ & $71(28.0)$ & $92(34.7)$ & 19 (30.6) & 0.59 \\
\hline Summer & 109 (13.4) & $9(12.7)$ & $20(12.3)$ & $38(15.0)$ & $32(12.1)$ & $10(16.1)$ & \\
\hline Autumn & $234(28.7)$ & $22(31.0)$ & $55(34.0)$ & $75(29.5)$ & $69(26.0)$ & $13(21.0)$ & \\
\hline Winter & $213(26.2)$ & $18(25.4)$ & $33(20.4)$ & $70(27.6)$ & $72(27.2)$ & $20(32.3)$ & \\
\hline
\end{tabular}

Abbreviations: BMI, body mass index; TV, television; PC, computer. ${ }^{a} x^{2}$-test and one-way ANOVA were used to test differences in proportions and means, respectively. 


\begin{tabular}{|c|c|c|c|c|c|c|c|c|}
\hline & \multirow[t]{2}{*}{ Total sample } & \multicolumn{3}{|c|}{ Midpoint of sleep } & \multirow[t]{2}{*}{$\mathrm{P}^{\mathrm{a}}$} & \multicolumn{2}{|c|}{ Sleep quality } & \multirow[t]{2}{*}{$P^{a}$} \\
\hline & & Early & Medium & Late & & Poor quality & Good quality & \\
\hline $\mathrm{N}(\%)$ & $814(100)$ & $278(34.2)$ & $265(32.6)$ & $271(33.3)$ & & $130(16.0)$ & $684(84.0)$ & \\
\hline \multicolumn{9}{|l|}{$\operatorname{Sex}(\mathrm{N}(\%))$} \\
\hline Men & $327(40.2)$ & $92(33.1)$ & $115(43.4)$ & $120(44.3)$ & 0.01 & $48(36.9)$ & $279(40.8)$ & 0.41 \\
\hline Women & $487(59.8)$ & $186(66.9)$ & $150(56.6)$ & $151(55.7)$ & & $82(63.1)$ & $405(59.2)$ & \\
\hline Age (years) & $48.0 \pm 15.4$ & $47.7 \pm 14.8$ & $47.5 \pm 14.6$ & $48.6 \pm 16.7$ & 0.69 & $51.4 \pm 16.3$ & $47.3 \pm 15.1$ & $<0.01$ \\
\hline \multicolumn{9}{|l|}{ BMI groups: $\mathrm{N}(\mathrm{N}(\%))$} \\
\hline Underweight & $18(2.2)$ & $8(2.9)$ & $7(2.6)$ & $3(1.1)$ & 0.36 & $3(2.3)$ & $15(2.2)$ & 0.20 \\
\hline Normal weight & $337(41.4$ & $110(39.6)$ & $116(43.8)$ & $111(41.0)$ & & $48(36.9)$ & $289(42.3)$ & \\
\hline Overweight & $296(36.3)$ & $111(39.9)$ & $91(34.3)$ & $94(34.7)$ & & $44(33.8)$ & $252(36.8)$ & \\
\hline Obese & $163(20.0)$ & 49 (17.6) & $51(19.2)$ & $63(23.2)$ & & $35(26.9)$ & $128(18.7)$ & \\
\hline BMI $\left(\mathrm{kg} / \mathrm{m}^{2}\right)$ & $26.5 \pm 5.1$ & $26.3 \pm 5.3$ & $26.3 \pm 4.7$ & $26.8 \pm 5.2$ & 0.49 & $27.7 \pm 6.6$ & $26.2 \pm 4.7$ & $<0.01$ \\
\hline \multicolumn{9}{|l|}{ Education (N (\%)) } \\
\hline$<12$ years & $609(78.8)$ & $223(80.2)$ & $195(73.6)$ & $191(70.5)$ & 0.26 & $107(82.3)$ & $502(73.4)$ & 0.05 \\
\hline$\geqslant 12$ years & $177(21.7)$ & $53(19.1)$ & $62(23.4)$ & $62(22.9)$ & & $20(15.4)$ & $157(23.0)$ & \\
\hline Missing & $28(3.4)$ & $2(0.7)$ & $8(3.0)$ & $18(6.6)$ & & $3(2.3)$ & $25(3.7)$ & \\
\hline \multicolumn{9}{|l|}{ Physical activity (N (\%)) } \\
\hline$\geqslant 2 \mathrm{~h} /$ week & $448(55.0)$ & $150(54.0)$ & $149(56.2)$ & $149(55.0)$ & 0.87 & $58(44.6)$ & $390(57.0)$ & $<0.01$ \\
\hline$<2 \mathrm{~h} /$ week & $366(45.0)$ & $128(46.0)$ & $116(43.8)$ & $122(45.0)$ & & $72(55.4)$ & $294(43.0)$ & \\
\hline TV and PC use (h/day) & $2.5 \pm 2.1$ & $2.4 \pm 1.8$ & $2.8 \pm 2.1$ & $3.3 \pm 2.4$ & $<0.01$ & $3.2 \pm 2.8$ & $2.7 \pm 1.9$ & $<0.01$ \\
\hline \multicolumn{9}{|l|}{ Smoking (N (\%)) } \\
\hline Current smoker & $217(26.7)$ & $65(23.4)$ & $60(22.6)$ & $92(33.9)$ & 0.02 & $45(34.6)$ & $172(25.1)$ & 0.02 \\
\hline Ex-smoker & $174(21.4)$ & $61(21.9)$ & $57(21.5)$ & $56(20.7)$ & & $32(24.6)$ & $142(20.8)$ & \\
\hline Non-smoker & $422(51.8)$ & $151(54.3)$ & $148(55.8)$ & $123(45.4)$ & & $53(40.8)$ & $369(53.9)$ & \\
\hline Missing & $1(0.1)$ & $1(0.4)$ & & & & & $1(0.1)$ & \\
\hline \multicolumn{9}{|l|}{ Season (N (\%)) } \\
\hline Spring & $258(31.7)$ & $81(29.1)$ & $97(36.6)$ & $80(29.5)$ & 0.13 & $39(30.0)$ & $219(32.0)$ & 0.44 \\
\hline Summer & 109 (13.4) & $42(15.1)$ & $24(9.1)$ & 43 (15.9) & & $15(11.5)$ & 94 (13.7) & \\
\hline Autumn & $234(28.7)$ & $79(28.4)$ & $81(30.6)$ & $74(27.3)$ & & $45(34.6)$ & $189(27.6)$ & \\
\hline Winter & $213(26.2)$ & $76(27.3)$ & $63(23.8)$ & $74(27.3)$ & & $31(23.8)$ & $182(26.6)$ & \\
\hline
\end{tabular}

Abbreviations: BMI, body mass index; PC, computer; s.d., standard deviation; TV, television. ${ }^{a} X^{2}$-test and one-way ANOVA were used to test differences in proportions and means, respectively. $P$-values showing significant differences in main characteristics by midpoint of sleep or sleep quality are in bold.

present study could not confirm other associations reported in the literature. ${ }^{19}$

Two recent studies among young Japanese women suggest that human chronotype is associated with intakes of certain nutrients and food. ${ }^{20,21}$ For instance, a late midpoint of sleep (evening type) was associated with higher energy intake from alcohol, fat, confectionary and meat. By contrast, the morning type was associated with a higher intake of, for example, calcium, vitamin $B_{6}$, vegetables and pulses. ${ }^{20}$ We could only find an association between early midpoint of sleep and higher intake of carbonated beverages. Compared with young Japanese women, men and women in our study population had an up to almost $3 \mathrm{~h}$ earlier midpoint of sleep in average, reflecting a completely different sleeping behaviour. As there was no specific instrument for human chronotype available in the BVS II, we used midpoint of sleep as a proxy. However, this measure is not able to distinguish between morning and evening types. Furthermore, we calculated the midpoint of sleep using information about weekday bedtimes and rise times. As reported previously, the midpoint of sleep may be different between weekdays and weekend day due to difference in daily routine between working days and free days. $^{28}$

Although subjects with a good sleep quality showed more favourable lifestyle habits than subjects with poor sleep quality did, we found that quality of sleep was not significantly associated with food, beverage, nutrients and energy intake in the present study. In contrast, among middle-aged female Japanese workers, poor sleep quality was associated with low intake of vegetables and fish and was associated with high intake of confectionary, noodles, energy drinks, sugar-sweetened beverages and carbohydrates. ${ }^{22}$ Data from NHANES showed that for example sleep maintenance difficulties were related to higher intake of salt and lower intake of carbohydrate and vitamin D. ${ }^{23}$

It is not surprising that short duration sleepers drank more coffee and caffeinated tea and therefore had a higher intake of caffeine. There is evidence that caffeine has a stimulating effect. The explanation of the association between sleep duration and other non-alcoholic beverages is not that simple. Different studies have shown that due to an increased feeling of hunger and appetite, subjects under sleep restriction consumed larger portions, more energy-dense foods and preferred foods high in fat or carbohydrates, compared with subjects with adequate sleep duration. ${ }^{6,9,12}$ The higher intake of carbonated beverages among short duration sleepers might be another example how energy and to a certain amount caffeine (for example, in coke energy drinks) could be supplied quickly. The explanation for a higher intake of tap water among short duration sleepers remains unclear. Although there is evidence about the association between sleep restriction and increased hunger and appetite, we found no study that examined an association of feeling thirsty under sleep restriction.

The association between sleep duration and intake of carbonated beverages has to be interpreted with cautiondue to the large number of non-consumers (69\%, data not shown). 


\begin{tabular}{|c|c|c|c|c|c|c|}
\hline \multirow{2}{*}{$\begin{array}{l}\text { Food intake } \\
\text { (g/day) }\end{array}$} & \multicolumn{6}{|c|}{ Sleep duration } \\
\hline & $<6 h \mathrm{n}=71$ & $6-6.5 h \mathrm{n}=162$ & $7 h \mathrm{n}=254$ & $7.5-8 h \mathrm{n}=265$ & $>8 h \mathrm{n}=62$ & $P^{b}$ \\
\hline Vegetables/potatoes & $207.7(180.0 ; 235.4)$ & $190.9(172.3 ; 209.6)$ & $203.8(187.9 ; 219.7)$ & $209.1(193.7 ; 224.5)$ & $193.1(162.8 ; 223.5)$ & 0.55 \\
\hline Fruits & $132.3(98.8 ; 165.7)$ & $133.8(111.8 ; 155.8)$ & $120.9(102.3 ; 139.5)$ & $133.5(115.3 ; 151.7)$ & $150.6(115.2 ; 186.1)$ & 0.59 \\
\hline Milk and dairy products & $161.2(122.7 ; 199.7)$ & $170.4(144.5 ; 196.3)$ & $168.9(146.9 ; 190.9)$ & $180.7(159.3 ; 202.1)$ & $200.2(158.0 ; 242.3)$ & 0.59 \\
\hline Bread & $115.2(101.9 ; 128.5)$ & $114.5(105.8 ; 123.2)$ & $117.1(109.7 ; 124.5)$ & $125.2(117.9 ; 132.4)$ & $118.8(104.7 ; 132.9)$ & 0.28 \\
\hline Cereals & $82.2(65.9 ; 98.5)$ & $68.6(57.9 ; 79.4)$ & $77.0(67.9 ; 86.1)$ & $75.2(66.3 ; 84.0)$ & 78.9 (61.6; 96.2) & 0.61 \\
\hline $\begin{array}{l}\text { Meat and processed } \\
\text { meat }\end{array}$ & $120.6(102.9 ; 138.4)$ & $116.1(104.2 ; 128.0)$ & $125.0(114.9 ; 135.2)$ & $115.3(105.4 ; 125.2)$ & $126.3(106.8 ; 145.7)$ & 0.54 \\
\hline Sugar and confectionary & $30.6(23.4 ; 37.9)$ & $32.3(27.4 ; 37.1)$ & $36.1(32.0 ; 40.3)$ & $31.4(27.3 ; 35.4)$ & $41.6(33.6 ; 49.5)$ & 0.08 \\
\hline Cakes & $44.4(31.2 ; 57.5)$ & $56.0(47.2 ; 64.9)$ & $53.6(46.0 ; 61.1)$ & $50.5(43.2 ; 57.8)$ & $48.5(34.1 ; 62.9)$ & 0.57 \\
\hline $\begin{array}{l}\text { Non-alcoholic } \\
\text { beverages }^{c}\end{array}$ & $2203.1(2033.1 ; 2373.0)$ & $1829.6(1715.2 ; 1943.9)$ & $1884.4(1787.2 ; 1981.6)$ & $1900.5(1806.0 ; 1995.1)$ & $1797.5(1611.2 ; 1983.8)$ & $<0.01$ \\
\hline $\begin{array}{l}\text { Fruit or } \\
\text { vegetable juice }\end{array}$ & $155.7(98.2 ; 213.2)$ & $184.3(145.4 ; 223.2)$ & $148.9(116.3 ; 181.4)$ & $174.3(142.5 ; 206.1)$ & $124.2(61.6 ; 186.8)$ & 0.36 \\
\hline $\begin{array}{l}\text { Carbonated } \\
\text { beverages }\end{array}$ & $157.1(91.7 ; 222.5)$ & $141.3(97.3 ; 185.2)$ & $92.9(55.5 ; 130.3)$ & $77.3(40.9 ; 113.6)$ & $149.2(77.5 ; 220.8)$ & 0.04 \\
\hline Water & $992.5(833.2 ; 1151.8)$ & $738.0(630.8 ; 845.2)$ & $912.0(820.9 ; 1003.2)$ & $877.0(788.4 ; 965.6)$ & 873.7 (699.0; 1048.3) & 0.04 \\
\hline $\begin{array}{l}\text { Coffee/ } \\
\text { black tea }^{\text {d }}\end{array}$ & $675.1(578.6 ; 771.6)$ & $598.8(533.4 ; 664.1)$ & $522.4(505.6 ; 577.0)$ & $559.0(505.6 ; 612.3)$ & $471.4(366.2 ; 576.5)$ & 0.01 \\
\hline Alcoholic beverages & $286.9(215.9 ; 358.0)$ & $256.8(209.0 ; 304.6)$ & $264.0(223.3 ; 304.6)$ & $286.5(247.0 ; 326.0)$ & $191.1(113.2 ; 269.0)$ & 0.23 \\
\hline Protein & $73.1(69.8 ; 76.4)$ & $70.3(68.1 ; 72.5)$ & $70.5(68.6 ; 72.4)$ & $70.6(68.8 ; 72.4)$ & $72.2(68.6 ; 75.8)$ & 0.55 \\
\hline Fat & $76.0(72.1 ; 79.9)$ & $80.6(77.9 ; 83.2)$ & $80.1(77.9 ; 82.4)$ & $77.9(75.8 ; 80.1)$ & $80.2(75.9 ; 84.5)$ & 0.16 \\
\hline Carbohydrate & $209.5(199.8 ; 219.2)$ & $203.7(197.2 ; 210.3)$ & $202.2(196.4 ; 207.8)$ & $207.0(201.6 ; 212.4)$ & $208.1(197.4 ; 218.8)$ & 0.52 \\
\hline $\begin{array}{l}\text { Energy } \\
\text { (kcal/day) }^{\mathrm{e}}\end{array}$ & $2061.0(1923.9 ; 2198.0)$ & $2031.8(1939.6 ; 2124.0)$ & $2025.7(1947.4 ; 2104.1)$ & $1989.0(1912.7 ; 2065.3)$ & $2065.9(1915.6 ; 2216.2)$ & 0.81 \\
\hline \multicolumn{7}{|c|}{$\begin{array}{l}\text { a Means were adjusted for age, sex, BMl, education, smoking, sport, TV/PC use, season and energy intake. }{ }^{b} P \text {-value resulting from F-test (Type III SS). }{ }^{\mathrm{C}} \text { Adjusted } \\
\text { mean of subjects with }<6 \mathrm{~h} \text { is significantly higher than adjusted means of subjects with } 6-6.5,7,7.5-8 \text { and }>8 \mathrm{~h}(P<0.05) .{ }^{\mathrm{d}} \text { Adjusted mean of subjects with } \\
<6 \mathrm{~h} \text { is significantly higher than adjusted means of subjects with } 7 \text { and }>8 \mathrm{~h}(P<0.05) .{ }^{e} \text { Means adjusted for age, sex, BMl, education, smoking, sport, TV/PC } \\
\text { use and season. }\end{array}$} \\
\hline
\end{tabular}

\begin{tabular}{|c|c|c|c|c|}
\hline \multirow[t]{2}{*}{ Food intake (g/day) } & \multicolumn{3}{|c|}{ Midpoint of sleep } & \multirow[t]{2}{*}{$\mathrm{P}^{\mathrm{b}}$} \\
\hline & Tertile 1 (early) $\mathrm{n}=278$ & Tertile 2 (medium) $\mathrm{n}=265$ & Tertile 3 (late) $\mathrm{n}=271$ & \\
\hline Vegetables/potatoes & $209.7(194.4 ; 225.0)$ & $199.3(183.7 ; 214.8)$ & $198.1(182.9 ; 213.3)$ & 0.44 \\
\hline Fruits & $126.1(108.1 ; 144.2)$ & $134.2(115.9 ; 152.5)$ & $131.9(113.9 ; 149.9)$ & 0.77 \\
\hline Milk and dairy products & $163.8(142.5 ; 185.0)$ & $170.6(149.1 ; 192.2)$ & $187.7(166.7 ; 208.7)$ & 0.22 \\
\hline Bread & $120.3(113.1 ; 127.5)$ & $120.4(113.1 ; 127.7)$ & $116.9(109.7 ; 124.1)$ & 0.71 \\
\hline Cereals & $78.8(70.1 ; 87.8)$ & $71.0(62.1 ; 79.9)$ & $75.0(66.2 ; 83.8)$ & 0.38 \\
\hline Meat and processed meat & $118.5(108.7 ; 128.3)$ & $123.9(113.9 ; 133.8)$ & $116.6(106.9 ; 126.3)$ & 0.51 \\
\hline Sugar and confectionary & $32.7(28.7 ; 36.7)$ & $32.5(28.4 ; 36.6)$ & $35.3(31.4 ; 39.3)$ & 0.50 \\
\hline Cakes & $49.1(41.8 ; 56.3)$ & $51.9(44.5 ; 59.2)$ & $55.0(47.8 ; 62.2)$ & 0.47 \\
\hline Non-alcoholic beverages & 1897.5 (1802.7; 1992.3) & $1899.8(1803.4 ; 1996.2)$ & $1898.2(1804.3 ; 1992.1)$ & 0.99 \\
\hline Fruit or vegetable juice & $158.6(126.9 ; 190.3)$ & $162.2(130.0 ; 194.4)$ & $170.5(139.3 ; 201.7)$ & 0.84 \\
\hline Carbonated beverages $^{c}$ & $144.1(108.0 ; 180.3)$ & $81.7(45.0 ; 118.5)$ & $96.5(60.7 ; 132.3)$ & 0.02 \\
\hline Water & $859.1(770.7 ; 947.6)$ & $891.0(801.1 ; 980.9)$ & $849.3(761.6 ; 936.9)$ & 0.76 \\
\hline Coffee/black tea & $555.6(502.1 ; 609.1)$ & $537.3(483.0 ; 591.6)$ & $585.2(532.5 ; 637.8)$ & 0.39 \\
\hline Alcoholic beverages & $237.1(197.8 ; 276.4)$ & $279.6(239.7 ; 319.5)$ & $283.8(244.9 ; 322.7)$ & 0.13 \\
\hline Protein & $71.3(69.5 ; 73.1)$ & $69.9(68.1 ; 71.8)$ & $71.2(69.4 ; 73.0)$ & 0.45 \\
\hline Fat & $80.0(77.9 ; 82.2)$ & $80.4(78.2 ; 82.6)$ & $77.3(75.1 ; 79.4)$ & 0.06 \\
\hline Carbohydrates & $205.7(200.3 ; 211.0)$ & $201.6(196.1 ; 207.1)$ & $207.7(202.4 ; 213.0)$ & 0.22 \\
\hline Energy (kcal/day) ${ }^{d}$ & $1976.4(1900.6 ; 2052.1)$ & $2016.8(1940.0 ; 2093.6)$ & $2064.3(1989.8 ; 2138.8)$ & 0.21 \\
\hline
\end{tabular}

Stratified analyses showed that sleep duration between nonconsumers and consumers of carbonated beverages differs significantly: the proportion of short duration sleepers is higher and therefore mean sleep duration is lower among nonconsumers. As consumers of carbonated beverages are significantly younger than non-consumers (mean age 40.3 years vs 51.4 years, $P<0.01$ ), there is also an age-effect. However, when intake of carbonated beverages is analysed in consumers only, short duration sleepers showed the highest intake amount. Indeed, there is a U-shaped association over the sleep duration 


\begin{tabular}{|c|c|c|c|}
\hline \multirow[t]{3}{*}{ Food intake (g/day) } & \multicolumn{2}{|c|}{ Sleep quality } & \multirow[t]{3}{*}{$\mathrm{P}^{\mathrm{b}}$} \\
\hline & Poor & Good & \\
\hline & $\mathrm{n}=130$ & $n=684$ & \\
\hline Vegetables/potatoes & $200.0(178.8 ; 221.1)$ & $202.7(191.9 ; 213.6)$ & 0.81 \\
\hline Fruits & $116.2(91.2 ; 141.3)$ & $133.5(120.6 ; 146.3)$ & 0.19 \\
\hline Milk and dairy products & $163.8(134.4 ; 193.2)$ & $176.5(161.4 ; 191.5)$ & 0.42 \\
\hline Bread & $114.0(104.0 ; 124.0)$ & $120.1(115.0 ; 125.2)$ & 0.25 \\
\hline Cereals without bread & $76.7(64.5 ; 89.0)$ & $74.7(68.4 ; 81.0)$ & 0.75 \\
\hline Meat and processed meat & 119.7 (106.2; 133.3) & $119.5(112.5 ; 126.4)$ & 0.97 \\
\hline Sugar and confectionary & $36.6(31.1 ; 42.2)$ & $33.0(30.1 ; 35.8)$ & 0.22 \\
\hline Cakes & $53.5(43.4 ; 63.5)$ & $51.8(46.6 ; 56.9)$ & 0.75 \\
\hline Non-alcoholic beverages & $1950.0(1819.2 ; 2080.9)$ & $1888.6(1821.5 ; 1955.7)$ & 0.38 \\
\hline Fruit or vegetable juice & $168.7(124.8 ; 212.5)$ & $163.1(140.7 ; 185.6)$ & 0.81 \\
\hline Carbonated beverages & $124.9(74.8 ; 175.1)$ & $104.2(78.5 ; 130.0)$ & 0.44 \\
\hline Water & 846.1 (723.9; 968.3) & 869.4 (806.7; 932.1) & 0.72 \\
\hline Coffee/black tea & $564.7(490.6 ; 638.7)$ & $559.7(521.8 ; 597.6)$ & 0.90 \\
\hline Alcoholic beverages & $248.6(194.2 ; 302.9)$ & $270.6(242.7 ; 298.5)$ & 0.45 \\
\hline Protein & $72.0(69.5 ; 74.5)$ & $70.6(69.3 ; 71.9)$ & 0.30 \\
\hline Fat & $80.4(77.4 ; 83.4)$ & $78.9(77.4 ; 80.5)$ & 0.36 \\
\hline Carbohydrates & $204.2(196.8 ; 211.7)$ & $205.3(201.4 ; 209.1)$ & 0.79 \\
\hline Energy $(\mathrm{kcal} / \text { day })^{c}$ & $2062.7(1958.3 ; 2167.1)$ & $2012.6(1959.1 ; 2066.0)$ & 0.37 \\
\hline
\end{tabular}

categories as the second highest intake of carbonated beverages was observed among subjects sleeping more than $8 \mathrm{~h}$. This holds true regardless of inclusion or exclusion of non-consumers. This result is also in line with findings from recent studies which showed that both short and long sleep duration is associated with the development of type 2 diabetes mellitus. ${ }^{29,30}$

A sensitivity analysis excluding underreporters (data not shown) revealed that sleep duration was still significantly associated with the intake of total non-alcoholic beverages and with carbonated beverages as well. The nature of these associations also remained the same as short duration sleepers $(<6 \mathrm{~h})$ showed the highest intake of non-alcoholic beverages in general and of carbonated beverages, long duration sleepers $(>8 \mathrm{~h})$ showed the second highest intake. Only the association between sleep duration and consumption of coffee/tea was no longer significant $(P=0.06)$. After the exclusion of under-reporters, midpoint of sleep remained significantly associated with carbonated beverages with highest intake among subjects with early midpoint of sleep. The lack of any association between sleep quality and dietary intake also remained when under-reporters were excluded.

One possible explanation for the lack of association between sleep duration and food, energy-providing nutrients and energy intake might be that compared with other study populations, our subjects differ in some sleep- and health-related characteristics. In different American studies, more than $35 \%$ of the subjects slept $6 \mathrm{~h}$ or less ${ }^{14,15,18}$ in contrast to $25.7 \%$ in our study. Also BMI differs considerably between these studies and our study, with a higher proportion of overweight or obese subjects in the U.S. samples. In our data, BMI was not significantly associated with sleep duration and midpoint of sleep and only slightly associated with PSQI, whereas subjects with poor sleep quality had a higher BMI than those with good sleep quality ( $27.1 \mathrm{vs} 26.2, P=0.05$ ). The results of energy intake also remained the same when the models were not adjusted for BMI.

Overall, the BVS II is a well-designed population-based study, representative for the Bavarian population to investigate dietary and lifestyle habits. Comprehensive data on dietary behaviour were available. In contrast to other studies, not only data on sleep duration was available but also for other sleep characteristics like midpoint of sleep as indicator of human chronotype and quality of sleep. With the PSQI a well-established instrument providing meaningful subjective information on different sleep characteristics was applied. However, the BVS II was originally not designed to detect associations with sleep characteristics why no objective measure of sleep was included and no other sleep-related information such as circadian rhythm variables or shift work was available. To take into account daylight duration, we used (astronomical) season as a proxy variable for daylight duration (which was not assessed in our study). We also tested meteorological seasons for adjustment. Both variables were not significantly associated with sleep duration, midpoint of sleep or sleep quality. As shown in a recent meta-analysis on seasonality of food intake, there is limited seasonal variation in consumption data at the aggregated food group level (as used in our analysis). ${ }^{31}$ Therefore we did not consider season or daylight duration in more depth.

Because of the cross-sectional study design, only associations could be described. The different sensitivity analyses showed that the results we found were robust. Besides the exclusion of underreporters, we additionally performed analyses stratified for sex, age groups ( $<40$ years, $40-<60$ years, $\geqslant 60$ years) and BMI groups $\left(<30 \mathrm{~kg} / \mathrm{m}^{2}, \geqslant 30 \mathrm{~kg} / \mathrm{m}^{2}\right)$. The results are available as Supplementary information (Supplementary Tables S1-S3). Although stratified analyses showed differences with regard to statistical significant associations, mainly beverage intake was associated with sleep and the overall patterns of intake amounts were comparable to those for the total population. For instance, highest intakes of total non-alcoholic beverages were found among subjects sleeping less than $6 \mathrm{~h}$ (even after stratification for sex, BMI groups or age). Except for the subjects aged 60 years and older, highest intake of coffee/black tea was also found among subjects sleeping less than $6 \mathrm{~h}$. Furthermore, the U-shaped association between sleep duration and carbonated beverages persisted for men, in subjects with BMl $<30 \mathrm{~kg} / \mathrm{m}^{2}$ and in subjects younger than 40 years. The highest intake of carbonated 
beverages (even when not significant) was found among subjects with early midpoint of sleep, irrespective of the strata.

\section{CONCLUSION}

In conclusion, our findings demonstrate few specific associations between sleep characteristics and dietary intake. Mainly sleep duration was associated with beverage intake. Midpoint of sleep was associated with carbonated beverage intake only, and quality of sleep was not related to food and beverage intake. Our results do not clearly support the hypothesis that dietary factors help to explain the mechanisms involved in sleep-disease associations. Only the association between carbonated beverages and sleep duration might be one example for the link between sleep and diabetes mellitus and obesity.

\section{CONFLICT OF INTEREST}

The authors declare no conflict of interest.

\section{ACKNOWLEDGEMENTS}

The preparation of this paper was supported by the DEterminants of Dlet and Physical ACtivity (DEDIPAC) Knowledge Hub. This work is supported by the Joint Programming Initiative 'Healthy Diet for a Healthy Life'. The funding agency of this work is the German Federal Ministry of Education and Research. We gratefully acknowledge the cooperation of the study participants as well as the work of al co-workers involved in the sampling of data. The BVS II was financially supported by funds from the Bavarian Ministry of Health and Consumer Protection, and the Kurt Eberhard Bode Foundation. CK is financially supported by the German Federal Ministry of Education and Research, Grant No. 01EA1372C.

\section{REFERENCES}

1 Choi KM, Lee JS, Park HS, Baik SH, Choi DS, Kim SM. Relationship between sleep duration and the metabolic syndrome: Korean National Health and Nutrition Survey 2001. Int J Obes 2008; 32: 1091-1097.

2 Xiao Q, Keadle SK, Hollenbeck AR, Matthews CE. Sleep duration and total and cause-specific mortality in a large US cohort: interrelationships with physical activity, sedentary behavior, and body mass index. Am J Epidemiol 2014; 180: 997-1006.

3 von Ruesten A, Weikert C, Fietze I, Boeing H. Association of sleep duration with chronic diseases in the European Prospective Investigation into Cancer and Nutrition (EPIC)-Potsdam study. PloS One 2012; 7: e30972.

4 Park SE, Kim HM, Kim DH, Kim J, Cha BS, Kim DJ. The association between sleep duration and general and abdominal obesity in Koreans: data from the Korean National Health and Nutrition Examination Survey, 2001 and 2005. Obesity 2009; 17: 767-771.

5 Gangwisch JE, Heymsfield SB, Boden-Albala B, Buijs RM, Kreier F, Pickering TG et al. Short sleep duration as a risk factor for hypertension: analyses of the first National Health and Nutrition Examination Survey. Hypertension 2006; 47: 833-839.

6 Hogenkamp PS, Nilsson E, Nilsson VC, Chapman CD, Vogel H, Lundberg LS et al. Acute sleep deprivation increases portion size and affects food choice in young men. Psychoneuroendocrinology 2013; 38: 1668-1674.

7 Markwald RR, Melanson EL, Smith MR, Higgins J, Perreault L, Eckel RH et al. Impact of insufficient sleep on total daily energy expenditure, food intake, and weight gain. Proc Natl Acad Sci USA 2013; 110: 5695-5700.

8 Brondel L, Romer MA, Nougues PM, Touyarou P, Davenne D. Acute partial sleep deprivation increases food intake in healthy men. Am J Clin Nutr 2010; 91: 1550-1559.

9 St-Onge MP, Roberts AL, Chen J, Kelleman M, O'Keeffe M, RoyChoudhury A et al. Short sleep duration increases energy intakes but does not change energy expenditure in normal-weight individuals. Am J Clin Nutr 2011; 94: 410-416.

10 Nedeltcheva AV, Kilkus JM, Imperial J, Kasza K, Schoeller DA, Penev PD. Sleep curtailment is accompanied by increased intake of calories from snacks. Am J Clin Nutr 2009; 89: 126-133.
11 Bosy-Westphal A, Hinrichs S, Jauch-Chara K, Hitze B, Later W, Wilms B et al. Influence of partial sleep deprivation on energy balance and insulin sensitivity in healthy women. Obes Facts 2008; 1: 266-273.

12 Spiegel K, Tasali E, Penev P, Van Cauter E. Brief communication: sleep curtailment in healthy young men is associated with decreased leptin levels, elevated ghrelin levels, and increased hunger and appetite. Ann Intern Med 2004; 141: 846-850.

13 Nedeltcheva AV, Kilkus JM, Imperial J, Schoeller DA, Penev PD. Insufficient sleep undermines dietary efforts to reduce adiposity. Ann Intern Med 2010; 153: 435-441.

14 Kant AK, Graubard BI. Association of self-reported sleep duration with eating behaviors of American adults: NHANES 2005-2010. Am J Clin Nutr 2014; 100: 938-947.

15 Grandner MA, Jackson N, Gerstner JR, Knutson KL. Dietary nutrients associated with short and long sleep duration. Data from a nationally representative sample. Appetite 2013; 64: 71-80.

16 Garaulet M, Ortega FB, Ruiz JR, Rey-Lopez JP, Beghin L, Manios Y et al. Short sleep duration is associated with increased obesity markers in European adolescents: effect of physical activity and dietary habits. The HELENA study. Int J Obes 2011; 35: 1308-1317.

17 Stern JH, Grant AS, Thomson CA, Tinker L, Hale L, Brennan KM et al. Short sleep duration is associated with decreased serum leptin, increased energy intake and decreased diet quality in postmenopausal women. Obesity (Silver Spring) 2014; 22: E55-E61.

18 Patterson RE, Emond JA, Natarajan L, Wesseling-Perry K, Kolonel LN, Jardack P et al. Short sleep duration is associated with higher energy intake and expenditure among African-American and non-Hispanic white adults. $J$ Nutr 2014; 144: $461-466$.

19 Dashti HS, Scheer FA, Jacques PF, Lamon-Fava S, Ordovas JM. Short sleep duration and dietary intake: epidemiologic evidence, mechanisms, and health implications. Adv Nutr 2015; 6: 648-659.

20 Sato-Mito N, Sasaki S, Murakami K, Okubo H, Takahashi Y, Shibata S et al. The midpoint of sleep is associated with dietary intake and dietary behavior among young Japanese women. Sleep Med 2011; 12: 289-294.

21 Sato-Mito N, Shibata S, Sasaki S, Sato K. Dietary intake is associated with human chronotype as assessed by both morningness-eveningness score and preferred midpoint of sleep in young Japanese women. Int J Food Sci Nutr 2011; 62: 525-532.

22 Katagiri R, Asakura K, Kobayashi S, Suga H, Sasaki S. Low intake of vegetables, high intake of confectionary, and unhealthy eating habits are associated with poor sleep quality among middle-aged female Japanese workers. J Occup Health 2014; 56: $359-368$

23 Grandner MA, Jackson N, Gerstner JR, Knutson KL. Sleep symptoms associated with intake of specific dietary nutrients. J Sleep Res 2014; 23: 22-34

24 Slimani N, Deharveng G, Charrondiere RU, van Kappel AL, Ocke MC, Welch A et al. Structure of the standardized computerized 24- $\mathrm{h}$ diet recall interview used as reference method in the 22 centers participating in the EPIC project. European Prospective Investigation into Cancer and Nutrition. Comput Methods Programs Biomed 1999; 58: 251-266.

25 Slimani N, Ferrari P, Ocke M, Welch A, Boeing H, Liere M et al. Standardization of the 24- hour diet recall calibration method used in the european prospective investigation into cancer and nutrition (EPIC): general concepts and preliminary results. Eur J Clin Nutr 2000; 54: 900-917.

26 Buysse DJ, Reynolds CF 3rd, Monk TH, Berman SR, Kupfer DJ. The Pittsburgh Sleep Quality Index: a new instrument for psychiatric practice and research. Psychiatry Res 1989; 28: 193-213.

27 Schofield WN. Predicting basal metabolic rate, new standards and review of previous work. Hum Nutr Clin Nutr 1985; 39(Suppl 1): 5-41.

28 Roenneberg T, Kuehnle T, Juda M, Kantermann T, Allebrandt K, Gordijn M et al. Epidemiology of the human circadian clock. Sleep Med Rev 2007; 11: 429-438.

29 Alnaji A, Law GR, Scott EM. The role of sleep duration in diabetes and glucose control. Proc Nutr Soc 2016; 1-9.

30 Kowall B, Lehnich AT, Strucksberg KH, Fuhrer D, Erbel R, Jankovic N et al. Associations among sleep disturbances, nocturnal sleep duration, daytime napping, and incident prediabetes and type 2 diabetes: the Heinz Nixdorf Recall Study. Sleep Med 2016; 21: 35-41.

31 Stelmach-Mardas M, Kleiser C, Uzhova I, Penalvo JL, La Torre G, Palys W et al. Seasonality of food groups and total energy intake: a systematic review and meta-analysis. Eur J Clin Nutr 2016; 70: 700-708. 